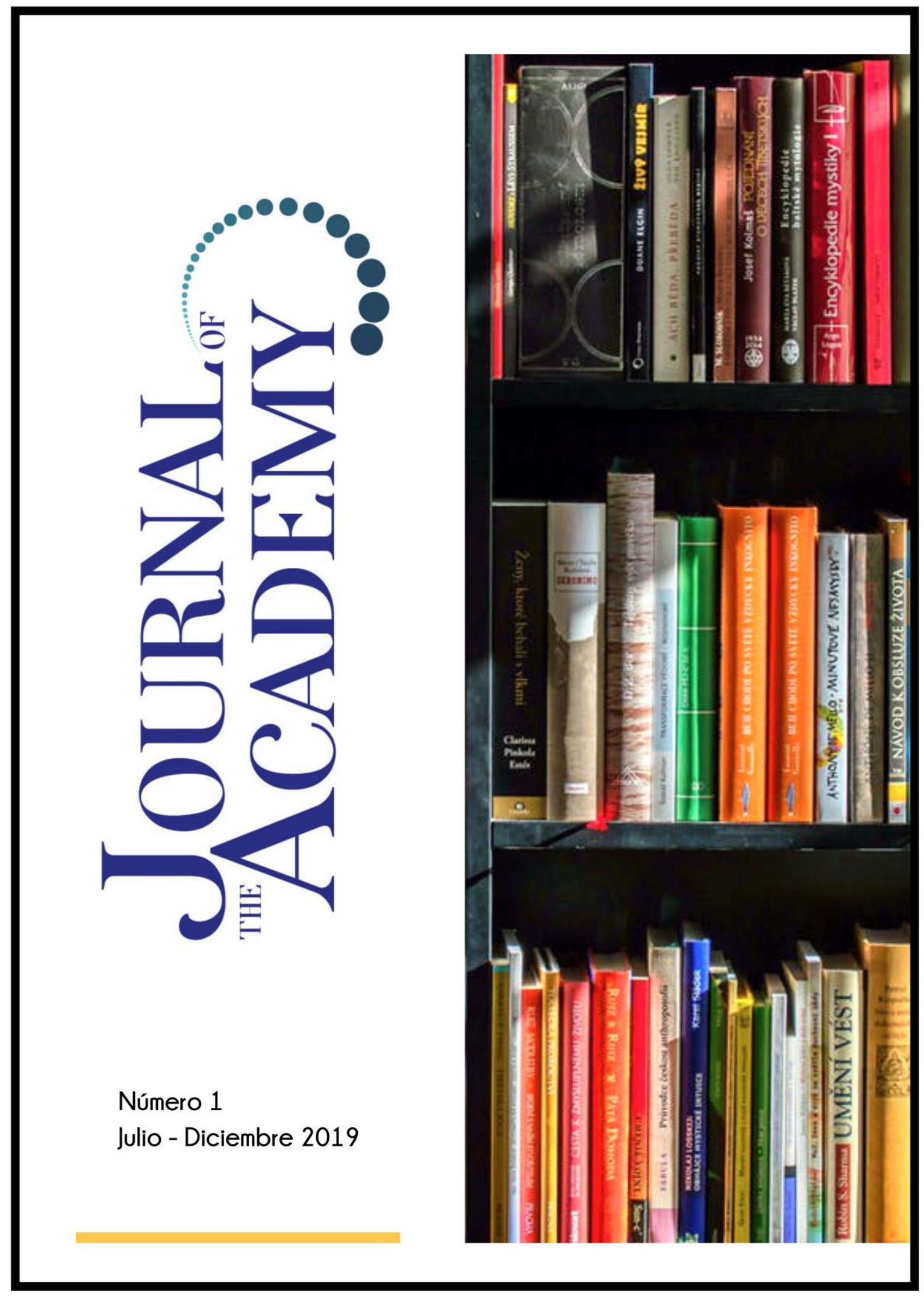




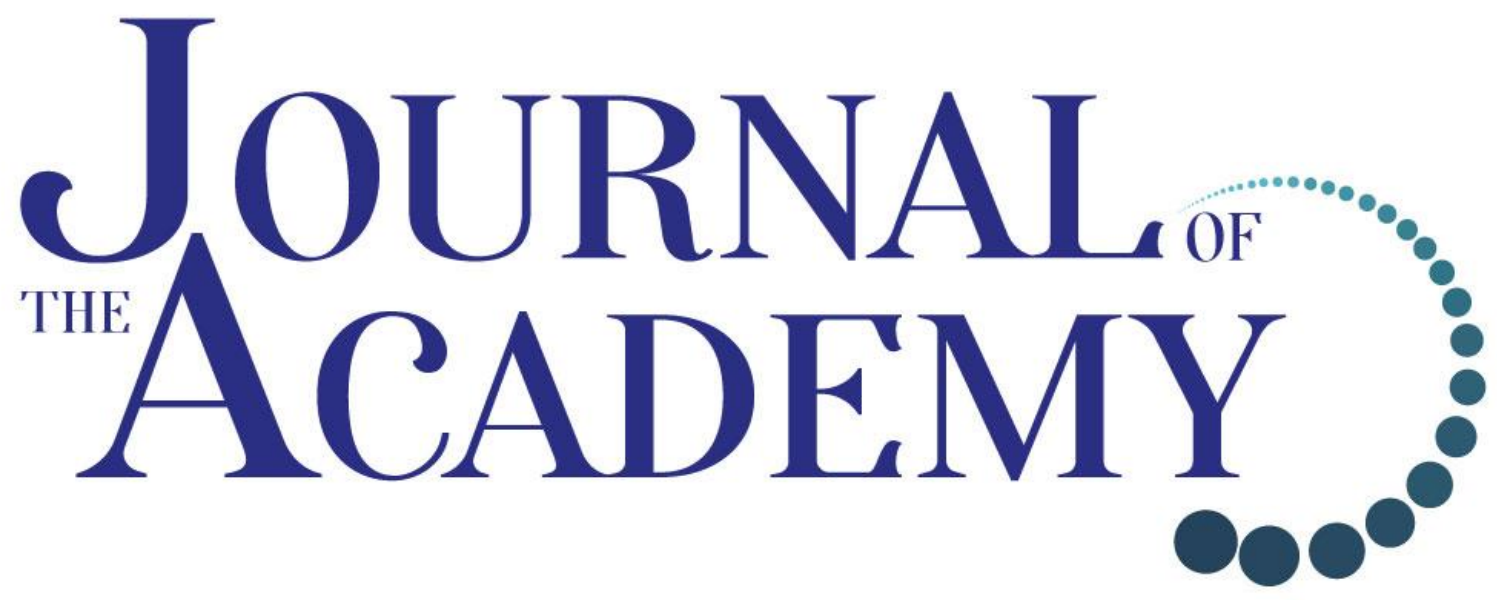

Publicación Científica de la Asociación de Universidades del Perú ASUP

Número 1

Julio-Diciembre 2019

www.journalacademy.org 


\section{ISSN 2707-0301}

\section{Directora-Editora en Jefe}

Ada Gallego Ruiz Conejo

Editor Científico

Oswaldo Orellana Manrique

Comité Científico

\section{Javier Carreón Guillén}

Universidad Nacional Autónoma de México, México Martino Contu

Universidad de Sassari, Italia

Jorge Enrique Elías Caro

Universidad del Magdalena, Colombia

Roberto Escalante Semerena

Universidad Nacional Autónoma de México, México

Oscar Ortega Arango

Universidad Autónoma de Yucatán, México

Alex Veliz Burgos

Universidad de Los Lagos, Chile

Comité Editorial

\section{Manuela Garau}

Centro Studi Sea, Italia

José Manuel González Freire

Universidad de Colima, México

Carlos Tulio da Silva Medeiros

Diálogos en Mercosur, Brasil

Eduardo Gomes Onofre

Universidade Estadual da Paraíba, Brasil

\section{Equipo Ejecutivo}

Juan Carlos Norabuena Castañeda

Thalia Chávez Cortéz

Journal Academy

Revista Semestral Open Access 


\title{
DIAGNÓSTICO DE LA INVESTIGACIÓN UNIVERSITARIA EN EL PERÚ
}

\section{DIAGNOSIS OF UNIVERSITY RESEARCH IN PERU}

Recibido: 26 de julio de 2018

Aceptado: 20 de mayo de 2019

\author{
Brenda Betzabe AGUILAR LÓPEZ \\ Universidad Nacional Mayor de San Marcos, Perú \\ brenda_aguilar990@hotmail.com \\ Carol Diana ARÉSTEGUI HUAMÁN \\ Universidad Nacional Mayor de San Marcos, Perú \\ canomecani@gmail.com \\ Cristina VERGARA COLLANTES \\ Universidad Nacional Mayor de San Marcos, Perú \\ catherincristi@gmail.com
}

\begin{abstract}
This research deals with reality and problems in which it is inserted the Peruvian University in its research policy, as gear essential for the development of a country. As main axes of study we analyze the financing, the role that plays the CONCYTEC and its relationship with the University; we reflect on the dichotomy of the field of research: ¿Humanities or natural sciences?, we analyze the problem in general and study data provided by the Scimago rankings on the universities of Peru, in the field of research. Finally, we arrive at the conclusions that serve as a basis for future studies.
\end{abstract}

Keywords: Diagnosis, research, University, financing, CONCYTEC, Scimago rankings, Law University No. 30220

Resumen: Esta investigación aborda la realidad y problemática en que está inserta la universidad peruana en su política de investigación, como engranaje indispensable para el desarrollo de un país. Como principales ejes de estudio analizamos el financiamiento, el rol que cumple el CONCYTEC y su relación con la universidad reflexionamos sobre la dicotomía del campo de la investigación: ¿ humanidades o ciencias naturales?, analizamos la problemática en general y estudiamos los datos proporcionados por el Ranking Scimago sobre las universidades del Perú, en materia de investigación. Por último, arribamos a las conclusiones que servirán de base para futuros estudios.

Palabras claves: Diagnóstico, investigación, universidad, financiamiento, CONCYTEC, Ranking Scimago, Ley Universitaria $\mathrm{N}^{\circ} 30220$

\section{Para Citar este Artículo:}

Aguilar López, Brenda Betzabe; Aréstegui Huamán, Carol Diana y Vergara Collantes, Cristina. Diagnóstico de la investigación universitaria en el Perú. Journal of Academy num 1 (2019): 44-56.

Licencia Creative Commons Atributtion Nom-Comercial 3.0 Uunported

(CC BY-NC 3.0)

Licencia Internacional

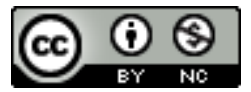




\section{Introducción}

Estamos viviendo tiempos vertiginosos en donde la ciencia es el motor del desarrollo. En este contexto las universidades son las que prioritariamente deben generar investigación, porque son centros de producción de conocimientos (claustros) en donde se forman los profesionales expertos en ciencia y tecnología, que coadyuvarán a la solución de los problemas básicos más elementales de la sociedad.

Para enfrentar los diversos retos que día a día demanda la sociedad, las universidades en el Perú tienen que superar el estilo escolástico de transmitir sólo información y qué estás se están encaminando por currículos basados en el desarrollo de competencias, capacidades y habilidades propias que requiere una carrera profesional. Instituciones, en donde la investigación y la docencia, deben conformar una unidad de acción para procurar que el estudiante interactúe con su propia realidad y la realidad nacional.

Pero si hacemos un diagnóstico, como en el presente artículo, el ideal de una universidad comprometida con el quehacer investigativo, se desvanece por múltiples factores: Austeros financiamientos, insensibilidad del estado, ineficiencia del capital humano al interior de la universidad y la ausencia de una cultura de la investigación. Los docentes, en su mayoría, solo ejercen la función de enseñar como trasmisión de información, sin entender que la investigación no es campo solo de privilegiados, sino que está al alcance de quien tenga voluntad de emprender búsquedas para solucionar problemas, y así justificar su actuación (presencia) en el mundo académico.

Ya lo ha manifestado Gary Becker, Premio Nobel de Economía: "el éxito o el fracaso económico de una nación ya no depende de la tecnología o de la maquinaria que posee, sino de lo que haga con su gente". Si nos alentamos con este pensamiento, diríamos que la universidad debe ser un crisol de gente crítica, investigadora, generadora de desarrollo y comprometido con el avance del desarrollo local, regional y nacional.

\section{Financiamiento}

La Ley Universitaria $N^{\circ} 30220$ (2014), en el artículo 3 define a la Universidad como una comunidad académica orientada a la investigación y a la docencia, que brinda una formación humanista, científica y tecnológica con una clara conciencia de nuestro país como realidad multicultural.

Siguiendo esta concepción, como la razón de ser de la Universidad, en el artículo 48, menciona que la investigación constituye una función esencial y obligatoria de la universidad, que la fomenta y realiza, respondiendo a través de la producción de conocimiento y desarrollo de tecnologías a las necesidades de la sociedad, con especial énfasis en la realidad nacional. En esta visión, los investigadores, PurizacaRosillo, Cardoza-Jiménez \& Herrera-Añazco, manifiestan que la producción científica de una institución depende de varios aspectos, siendo el financiamiento un factor importante, ya que posibilita realizar investigaciones más complejas o publicar en revistas de mayor impacto que en algunos casos requieren pago por publicación.

El análisis que se realiza en esta parte del artículo, está relacionado al presupuesto asignado para la investigación en las universidades. Después de tres años de aprobado la Ley Universitaria, los avances en la producción de investigación en las Universidades Públicas, sobre todo en la Universidad Nacional Mayor de San Marcos, Universidad Nacional de Ingeniería y la Universidad Nacional Agraria de la Molina ${ }^{1}$, siendo éstas de mayor jerarquía, no han sido los más óptimos, ya que el financiamiento aún no han cubierto las expectativas que se esperaba. Para mayor visualización, presentamos la tabla $\mathrm{N}^{\circ} 1$ de distribución del presupuesto en investigaciones científicas.

\footnotetext{
${ }^{1}$ Según Scopus, son las tres universidades públicas que tienen mayores investigaciones científicas.
} 
Tabla $\mathrm{N}^{\circ} 1$

\begin{tabular}{|l|l|l|l|}
\hline UNIVERSIDADES & $\mathbf{2 0 1 5}$ & $\mathbf{2 0 1 6}$ & $\mathbf{2 0 1 7}$ \\
\hline Universidad Nacional Mayor de San Marcos & $12,363,426$ & $14,963,432$ & $20,624,000$ \\
\hline Universidad Nacional de Igeniería & $6,021,981$ & $12,998,042$ & $12,998,042$ \\
\hline Universidad Nacional Agraria de la Molina & $3,892,903$ & $7,186,213$ & $7,186,213$ \\
\hline
\end{tabular}

Fuente: Amigable-Ministerio de Economía y Finanzas

Elaboración propia

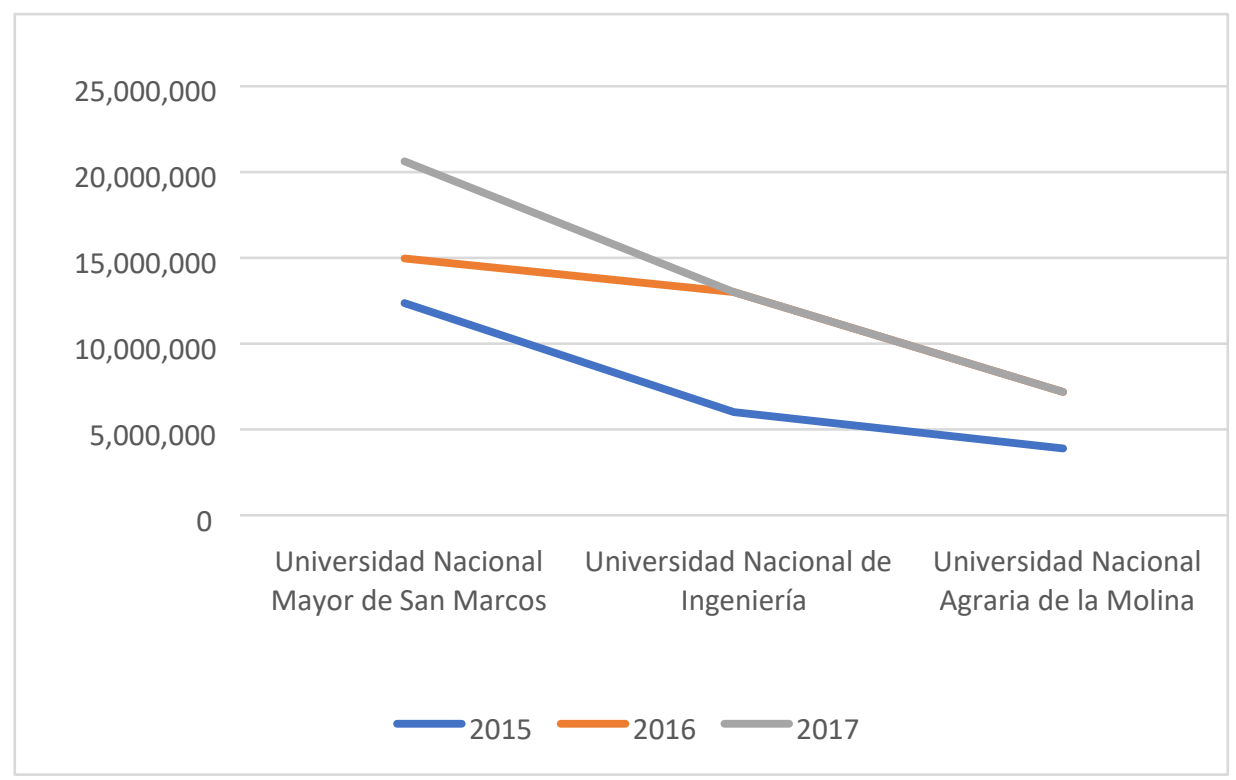

Gráfico 1

Según lo presentado en la Tabla $N^{\circ}$ 1, la Universidad Nacional Mayor de San Marcos cuenta con un mayor presupuesto y que ha evidenciado un notable incremento en el presupuesto para el año 2017, sino que (pero esto) se debe a que del total del presupuesto sólo S/. $6333,704.00$ provienen de recursos propios y por donaciones, la diferencia son los recursos del tesoro público.

Para el caso de la UNI y la Universidad Nacional Agraria de la Molina, los recursos que les corresponden del tesoro público son S/ 2 109,566.00 y S/ 3 100,637.00 respectivamente. Por lo tanto, la diferencia de sus presupuestos proviene de recursos que cada universidad generan y por donaciones.

Ahora bien, con los datos presentados se puede evidenciar que las distribuciones del presupuesto, no calza con lo mencionado en la Ley Universitaria, ya que son las universidades públicas las que tienen que buscar su propio financiamiento para que puedan cubrir todos los gastos que les genera poder realizar una investigación y así seguir manteniéndose en el ranking de las mejores universidades que realizan investigaciones para mantenerse a la vanguardia.

Como parte de esta realidad, se puede mencionar que la CONCYTEC ${ }^{2}$, dejó de ofrecer gratuitamente hasta el 2016 el acceso a las bases de datos ScienceDirect y Scopus, debido a la falta de financiamiento del gobierno central lo que ha causado el cierre de estas plataformas para los peruanos que quieran realizar investigaciones (rpp noticias 2016).

La priorización de gastos que realice el Gobierno Central, no siempre concuerda con las necesidades que puedan tener las Universidades Públicas, es por ello cuando la UNESCO realiza sus evaluaciones, el Perú siempre se encuentra como uno de los países que menos invierte en investigaciones.

\footnotetext{
${ }^{2}$ Consejo Nacional de Ciencia, Tecnología e Innovación Tecnológica
} 
Para cambiar esta realidad, que asfixia sobremanera a las Universidades y generen un aporte a la sociedad, se debe dar las facilidades para que puedan disponer de una auténtica autonomía económica, que les permita disponer de los recursos destinados a la ejecución de sus necesidades prioritarias sin dejar de lado la razón de ser de una universidad comprometida con el desarrollo del país.

\section{EI CONCYTEC, ente rector}

EI CONCYTEC estaría reconocido por su liderazgo y capacidad para orientar el desarrollo científico, tecnológico y de innovación en el Perú: y aporta significativamente en la toma de decisiones que contribuyen al desarrollo sostenible. Por ser una institución que estaría coadyuvando al desarrollo de la investigación universitaria en el Perú, debemos estudiar su grado de eficiencia y contribución, y detectar su problemática en el cumplimiento de sus funciones. Para ello primeramente tenemos que conocer su naturaleza.

Entre sus principales funciones podemos mencionar que se encarga de formular la política y planes nacionales de desarrollo científico y tecnológico, articulando las propuestas sectoriales, regionales e institucionales de Ciencia y Tecnología (CTel), con los planes de desarrollo socioeconómico, ambiental y cultural, entre otros del país; y formula y propone el Programa Nacional de Cooperación Técnica y Financiera Internacional para la CTel, en coordinación con el Ministerio de Economía y Finanzas, y la Agencia Peruana de Cooperación Internacional, a fin de establecer directivas y políticas vinculantes para el sector público y orientadoras para el sector privado, en el ámbito del Sistema Nacional de Ciencia, Tecnología e Innovación.

\section{Relación con la universidad}

EL CONCYTEC en su Reglamento de calificación y registro de investigadores en Ciencia y Tecnología, en el artículo 4, define al docente investigador universitario como una persona que se dedica a la generación de conocimiento e innovación, a través de la investigación, y que éste, para ser reconocido como tal, debe estar inscrito al Registro Nacional de Investigadores en Ciencia y Tecnología-REGINA, que es el Registro de personas naturales que poseen capacidades, establecidas de acuerdo a una calificación, para realizar labores de investigación científica y/o desarrollo tecnológico, y también debe completar su registro en la plataforma virtual del DINA- Directorio General de Investigadores e Innovadores.

Asimismo, el docente universitario para ser considerado investigador tiene que reunir los siguientes requisitos:

a) Grado de Bachiller, Maestro o Doctor, o título profesional.

b) Publicaciones en revistas científicas indexadas.

c) Publicación de libros y/o capítulos de libros o edición de libros de su especialidad.

d) Registro de propiedad intelectual como patentes u otras modalidades de protección de invenciones o nuevas tecnologías.

e) Asesoramiento de tesis sustentadas de bachillerato, título profesional, maestría y/o doctorado.

f) Valor del índice h de Scopus.

g) Experiencia en proyectos de investigación científica y/o desarrollo tecnológico.

h) Ponencias en congresos, seminarios u otros eventos de su especialidad a nivel nacional y/o internacional.

Por otro parte, en el artículo 8 de su reglamento, señala que el docente investigador debe ser evaluado en relación con su excelencia académica, la categoría establecida en la Ley Universitaria:

Otro punto que es importante destacar es en cuanto a los proyectos aprobados por CONCYTEC cuyas prioridades a las postulaciones que se presenten en concurso público deben responder a las prioridades de este y son las siguientes: Ciencias Básicas (Biología, Física, Matemática y Química). 


\section{Ciencias naturales versus humanidades}

Como podemos observar, las líneas de investigación que prioriza CONCYTEC son en Ciencias Básicas. En la página de Regina, donde se encuentran la mayor cantidad de investigadores registrados, en su mayoría son de campos aplicados a las ciencias de la salud o ingeniería. Ante esta realidad surge la necesidad de preguntarse: ¿Qué pasa con las investigaciones en el campo de las humanidades? Al respecto se ha desinformado bajo la creencia, muy extendida, de que las humanidades no tienen aplicación práctica, es decir, que no son útiles. En tal sentido, las ciencias, la tecnología sí tendrían utilidad, ya que explican y resuelven problemas, y se verifica su importancia mediante prácticas reales y objetivas, y sus resultados son cuantificables.

Como defensa argüimos que las humanidades sí son útiles, por ejemplo, para sentar las bases de una ciudadanía democrática. Esta condición es posible gracias al cultivo de un pensamiento crítico y creativo, al reconocimiento de la diversidad, la empatía con otras experiencias humanas y la reflexión sobre la complejidad del mundo. Literatura, filosofía, historia, psicología, ética y artes forman el pensar y el sentir sobre los otros y nos ayudan a comprender que las diferencias son un desafío para el consenso, mas no una muralla infranqueable. Esta creencia falsa es un resabio de la vieja dicotomía entre ciencias naturales y ciencias del espíritu establecida por Dilthey, quien estimaba que la esencia de aquellas residía en la explicación y de estas en la interpretación. Ambas facultades intelectuales fueron distorsionadas a favor de una sobreestimación de las ciencias naturales en perjuicio de las humanidades y las ciencias sociales. La superioridad de la explicación sobre la interpretación se fundamentó en la necesidad de alcanzar resultados constatables y universalizables, lo cual implicaba reducir al máximo las apreciaciones evaluativas y especulativas. El giro lingüístico, desde Wittgenstein hasta el postestructuralismo, ha insistido en la condición primaria de la comprensión analítica del lenguaje antes de iniciar cualquier reflexión sobre la realidad humana, social o natural. Por consiguiente, si el lenguaje organiza nuestra representación de la realidad, las humanidades tienen mucho que decir al respecto.

"De acuerdo, pero las humanidades no son rentables", es también una objeción frecuente. Mientras las áreas de ciencia y tecnología reciben ingentes presupuestos para investigación, becas, pasantías, etc., por el contrario, las humanidades, salvo excepciones notables, no están contempladas dentro de las áreas estratégicas para el desarrollo nacional. CONCYTEC, la institución rectora del sistema nacional de investigación científica en el Perú ha establecido áreas estratégicas a las cuales se destinan fondos para becas de posgrado en universidades nacionales e internacionales: no se incluyen maestrías ni doctorados en humanidades ni en ciencias sociales. En Argentina, antes del recorte determinado por el gobierno de Mauricio Macri, Conicet otorgaba becas doctorales y posdoctorales para investigaciones en ciencia, tecnologías, ciencias sociales y humanidades. El mayor recorte lo han sufrido las humanidades. El argumento es que la necesidad de obtener resultados tangibles es impostergable; en consecuencia, impactan directamente en la sociedad debido a que se las necesita. De esto infieren que, en los casos más logrados, la inversión es retornable.

Desde otras posiciones se afirma que las humanidades carecen de actualidad en el siglo XXI, argumento sucedáneo del anterior. Así, se coloca a las humanidades por debajo de la ciencia y la tecnología, circunstancia que replica la oposición ciencias/humanidades o artes/tecnología, por lo cual se estima que su decir sobre el presente es limitado. Pensemos brevemente en Julio Verne, Arthur C. Clarke, Ray Bradbury, Isaac Asimov, Geoge Orwell o Aldous Huxley y examinemos con claridad si realmente las letras no tendrían que decirnos mucho sobre el presente y el futuro, inclusive. La ciencia ficción ha mostrado ser tanto o más política que la narrativa realista. Y, aún supuesta la inocuidad contemporánea de las humanidades, ¿por qué la historia reciente del totalitarismo da cuenta de su obsesión por seducir o controlar a los intelectuales y sus discursos? ¿Por qué tanta insania contra libros que carecen de vigencia y cuyos contenidos palidecen ante el vigor de la tecnología? ¿Qué motiva a un Estado represor a prohibir la circulación de libros de psicoanálisis, sociología y filosofía, retirarlos de las bibliotecas, quemarlos públicamente y perseguir, asesinar o exiliar a sus autores? Pues el dominio sobre un discurso al que, en realidad, se teme por su aliento subversivo. (Artículo de opinión, Carlos Arturo Caballero: Mitos, sobre las humanidades) 


\section{Problemática detectada}

El CONCYTEC, ha detectado la problemática en la generación del conocimiento científico, tanto a nivel universitario, como en general, que nos permitimos exponer, en síntesis:

a.- Los programas de formación, con orientación a la investigación, que ofrecen las universidades no están alineados con las necesidades sociales, económicas y ambientales y presentan escasos aportes a la solución de los problemas de dicho sector.

b.- Los centros de investigación y desarrollo tecnológico, además de sus debilidades en infraestructura y equipamiento, presentan poca vinculación con las necesidades sociales, económicas y ambientales, dado que realizan proyectos de investigación y desarrollo con bajos niveles de aplicación práctica y, por lo tanto, con poca demanda en el sector empresarial.

c.- La protección de la propiedad intelectual en el Perú es un tema que se encuentra rezagado. El sistema de protección de la propiedad intelectual es débil y desprotege la capacidad inventiva de los actores generadores de conocimiento tecnológico.

d.- El Perú no cuenta con un sistema adecuado para atraer y retener talentos y menos para quienes están relacionados con la actividad científica.

Tabla $\mathrm{N}^{\circ} 2$ que presenta datos que corroboran el déficit de científicos:

\begin{tabular}{|c|c|c|c|c|}
\hline \multicolumn{5}{|c|}{$\begin{array}{l}\text { INVESTIGADORES CON GRADO DE DOCTOR REQUERIDOS POR CAMPO DE ESPECIALIZACION } \\
\text { (Nümero de investigadores y graduados) }\end{array}$} \\
\hline Especialidad & $\begin{array}{l}\text { Doctores } \\
\text { investigadores } \\
\text { actual }\end{array}$ & $\begin{array}{c}\text { Doctores } \\
\text { investigadores } \\
\text { optimo }\end{array}$ & $\begin{array}{c}\text { Brecha de } \\
\text { doctores } \\
\text { Invetigadores }\end{array}$ & $\begin{array}{l}\text { Brecha de } \\
\text { doctores } \\
\text { graduados }\end{array}$ \\
\hline Ciencias Naturales & 550 & 3383 & 2833 & 4047 \\
\hline Ingenieria y Tecnologia & 527 & 5349 & 4822 & 6889 \\
\hline Ciencias Médicas y de la Salud & 262 & 2555 & 2293 & 3275 \\
\hline Ciencias Agricolas & 177 & 1913 & 1736 & 2479 \\
\hline Subtotal & 1516 & 13200 & 11684 & 16691 \\
\hline Ciencios Sociaies & 254 & 3129 & 2875 & 4107. \\
\hline Humanidades & 78 & 1201 & 1123 & 1604 \\
\hline Subtotal & 332 & 4330 & 3998 & 5711 \\
\hline Total & 18.48 & 17529 & 15681 & 22.402 \\
\hline
\end{tabular}

a.- Los salarios y asignaciones económicas son limitados y el nivel y prestigio de los centros no constituyen incentivos que atraigan talentos.

b.- En términos generales, la calidad de la educación en el Perú es una de las más bajas en Latinoamérica, debido a múltiples factores, entre ellos: los centros de formación no cuentan con una masa crítica de docentes con niveles elevados de calidad, la infraestructura y el equipamiento son inadecuados.

c.- El número de programas de formación superior (maestrías y doctorados), especialmente los relacionados a ciencia y tecnología, es reducido. A pesar de haber aumentado significativamente el número de universidades e institutos tecnológicos, estos están concentrados en carreras poco vinculadas a la ciencia y tecnología.

d.- La educación técnica en el Perú cuenta con bajo nivel de calidad académica, la infraestructura es deficiente y los contenidos académicos no están alineados con las necesidades del país en materia de ciencia, tecnología e innovación tecnológica. 
e.- Los resultados de exámenes internacionales de rendimiento escolar dan cuenta del bajo nivel que presenta la educación básica peruana. Esta situación impacta negativamente en las posibilidades que tienen los estudiantes para acceder a la educación superior de calidad.

f.- A esta problemática se suma el poco manejo de otros idiomas, en especial el inglés, que limita el acceso a publicaciones académicas de calidad y a los avances en investigación y generación de conocimiento.

\section{Fondo Nacional de Desarrollo Científico, Tecnológico y de Innovación Tecnológica}

Para centrarnos mejor diremos que la unidad encargada de verificar los procesos de financiamiento de proyectos de investigación se llama Fondo Nacional de Desarrollo Científico, Tecnológico y de Innovación Tecnológica (FONDECYT), y entre sus principales funciones encontramos: Calificar la viabilidad económico-financiera de los proyectos de Ciencia, Tecnología e Innovación; intervenir en el financiamiento de los programas y proyectos regionales de Ciencia, Tecnología e Innovación; suscribir convenios sobre estudios y proyectos de investigación e innovación tecnológica con regiones y universidades; disponer fondos para el financiamiento de proyectos de ciencias básicas; otorgar donaciones, subsidios y financiar becas; conceder financiamientos directos e indirectos (fianzas, garantías y avales)

Para entender y procesar este financiamiento, analizamos el documento sobre el I Censo Nacional de Investigación y Desarrollo 2016, donde se encuentra el informe del Gasto Interno en Investigación y Desarrollo por sector institucional, lo cual evidenció que el 2014, el gasto de las universidades representó 43.2 por ciento del gasto total, cifra que aumentó a 46.8 por ciento del gasto total en el año 2015 . Si bien este importante incremento se explica por el mayor gasto en I+D de las universidades públicas, son las universidades privadas sin fines de lucro quienes tienen una mayor participación en ambos años, con alrededor de 30 por ciento.

\begin{tabular}{|c|c|c|c|c|}
\hline \multirow{2}{*}{ Sector Institucional } & \multicolumn{2}{|l|}{2014} & \multicolumn{2}{|l|}{2015} \\
\hline & Millones de SI & $\%$ & Millones de $\mathbb{S}$ & $\%$ \\
\hline Institutos Públicos de Investigación & 194.7 & 44.4 & 215.4 & 41.6 \\
\hline Instituto Público de Investigación & 184.2 & 42.0 & 202.1 & 39.0 \\
\hline Instituto de Salud & 10.5 & 2.4 & 13.3 & 2.6 \\
\hline Universidad & 189.3 & 43.2 & 242.1 & 46.8 \\
\hline Universidad pública & 58.2 & 13.3 & 81.0 & 15.7 \\
\hline Universidad privade sin fines de lucro $1 /$ & 125.4 & 28.6 & 145.2 & 28.0 \\
\hline Universidad privada con fines de lucro $2 /$ & 5.7 & 1.3 & 15.9 & 3.1 \\
\hline Institución Privada Sin Fines de Lucro & 53.2 & 12.2 & 59.2 & 11.4 \\
\hline Otro $3 /$ & 0.9 & 0.2 & 0.8 & 0.2 \\
\hline Total & 438.1 & 100.0 & 517.5 & 100.0 \\
\hline
\end{tabular}

1/ Universidades que tienen una organización jurídica de Asociación.

2/ Universidades que tienen una organización jurídica de Sociedades.

3/ Otro está conformado principalmente por sociedades anónimas que se dedican exclusivamente a realizar investigación.

Fuente: I Censo Nacional de Investigación y Desarrollo.

Elaboración: CONCYTEC - Dirección de Investigación y Estudios.

Tabla 3

Asimismo, se destaca el tipo de investigaciones que se realizaron durante el período 2014-2015, en donde se destaca que gran parte de este gasto estuvo dirigido hacia la investigación aplicada, seguida por la investigación básica y el desarrollo tecnológico. 


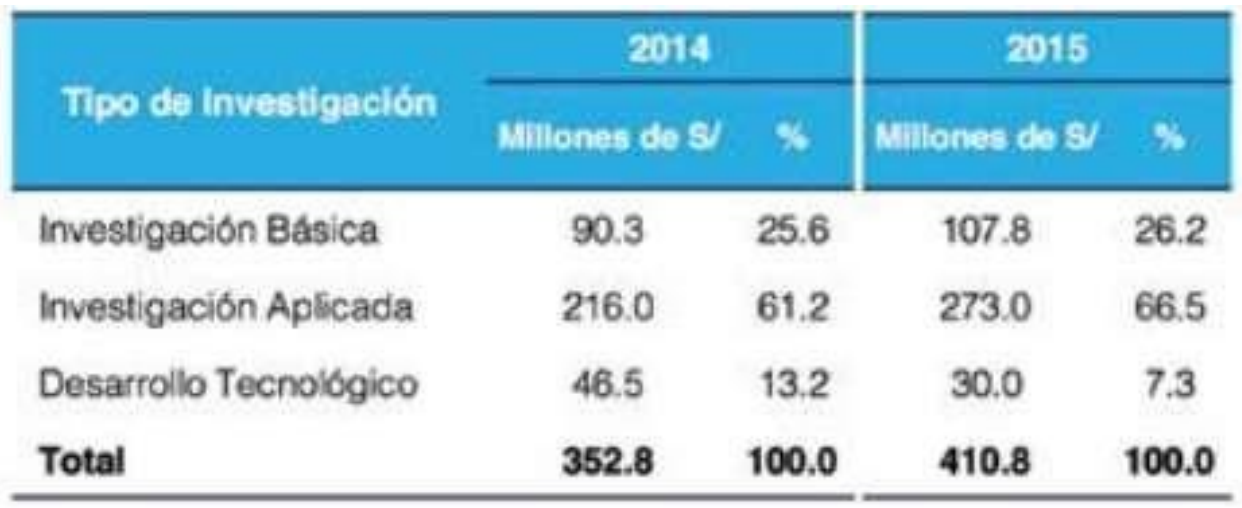

Fuente: I Censo Nacional de Investigación y Desarrollo.

Elaboración: CONCYTEC - Dirección de Investigación y Estudios.

Por otra parte, los institutos públicos de investigación destinaron un mayor porcentaje de su gasto corriente a la investigación aplicada, que fue alrededor de 75 por ciento, siendo menor la distribución en la investigación básica y desarrollo tecnológico. Las instituciones privadas sin fines de lucro también desviaron el mayor porcentaje de su gasto corriente a actividades relacionadas a la investigación aplicada, además se resalta el escaso presupuesto destinado a actividades de desarrollo tecnológico. Asimismo, las universidades privadas sin fines de lucro presentaron una repartición más equitativa entre investigación básica y aplicada, aunque se resalta la fuerte caída en la participación del gasto corriente en desarrollo tecnológico para el año 2015.

\begin{tabular}{|c|c|c|c|c|c|c|}
\hline Total & 25.6 & 61.2 & 13.2 & 26.2 & 66.5 & 7.3 \\
\hline $\begin{array}{l}\text { Instituto público de } \\
\text { investigación 1/ }\end{array}$ & 11.6 & 77.0 & 11.4 & 11.2 & 75.8 & 13.1 \\
\hline Universidad pública & 24.2 & 70.9 & 4.9 & 24.1 & 69.2 & 6.7 \\
\hline $\begin{array}{l}\text { Universidad privada sin fines } \\
\text { de lucro }\end{array}$ & 44.4 & 34.3 & 21.3 & 45.7 & 52.5 & 1.9 \\
\hline $\begin{array}{l}\text { Universidad privada con fines } \\
\text { de lucro }\end{array}$ & 42.9 & 30.7 & 26.4 & 55.4 & 26.2 & 18.4 \\
\hline $\begin{array}{l}\text { Institución privada sin fines de } \\
\text { lucro }\end{array}$ & 21.1 & 75.8 & 3. & 21.1 & 76.8 & 2.1 \\
\hline \multirow[t]{2}{*}{ Otro 2} & 51.0 & 20.8 & 28.1 & 55.2 & 21.6 & 23.2 \\
\hline & \multicolumn{3}{|c|}{2014} & \multicolumn{3}{|c|}{2015} \\
\hline - Investigación Básice & \multicolumn{3}{|c|}{ = Investigacion Aplicada } & \multicolumn{3}{|c|}{ = Desarrollo Tecnologico } \\
\hline
\end{tabular}

Los gastos destinados por área de conocimiento se clasifican de acuerdo a la participación según las especialidades que más demanda han obtenido en el período 2014-2015. El primer lugar lo tienen las Ciencias naturales, Ingeniería y tecnología, Ciencias sociales, Ciencias agrícolas, Ciencias médicas y de la Salud; finalmente se encuentran las Humanidades. 


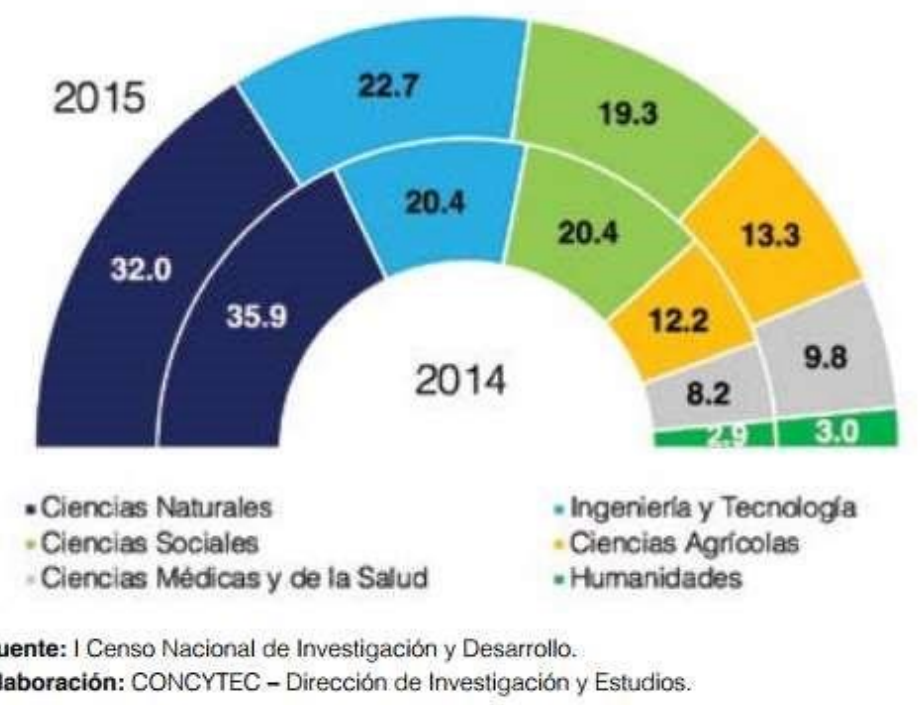

La evaluación presentada en el Censo, muestra la distribución del gasto de los recursos públicos a cada institución de Educación Superior, lo que evidencia la preocupación por la investigación y su aplicación en la sociedad.

En cuanto al presupuesto destinado para las Universidad privadas que representa un porcentaje mayor, se debe a que ellas cuentan con el mayor despliegue de presentaciones de proyectos de investigación a los concursos, ya que cuentan con las condiciones necesarias para realizarlas. Por otra parte, las Universidades Públicas, si bien cuentan con los profesionales idóneos, tienen una gran muralla en lo contemplado en la Ley Universitaria y en las normativas restrictivas aplicadas por el Ministerio de Economía y finanzas.

Destacamos también que el $77.1 \%$ de los fondos con los que se financian los gastos en Perú en Investigación y Desarrollo (I+D) provienen de fondos nacionales, mientras que el resto (22.9\%) proviene de fuentes extranjeras, así lo reveló el I Censo Nacional de Investigación y Desarrollo a Centros de Investigación 2016.

\begin{tabular}{|c|c|c|c|c|}
\hline \multirow{2}{*}{ 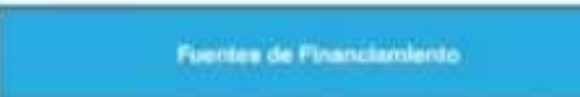 } & \multicolumn{2}{|l|}{2018} & \multicolumn{2}{|l|}{2015} \\
\hline & Milonect dis $\mathbf{8}$ & $\mathbf{v}$ & Milibenes de $y$ & 8 \\
\hline Fondos propise det Caintro de imealigación o instivción & 253.5 & 57.9 & 3019 & 563 \\
\hline Totid de Fondos proverientes os terceros & 754 & 172 & 97.1 & $18 \mathrm{~B}$ \\
\hline Fonoss pietion comorsaties & 28 & eet & 32 & ab \\
\hline Finsse de encruate: & as & 167 & $\omega$ & ne.e \\
\hline 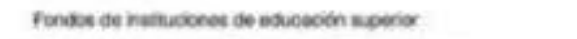 & rs & 0.7 & a. & $* 7$ \\
\hline 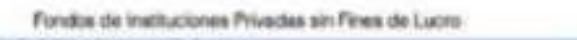 & as & ex & zs & as \\
\hline Total Fondos Nacionales & 222.9 & TS.1 & 300.9 & $n .1$ \\
\hline Donacionis extrarieras y fondos coneurabias & 867 & 19.8 & 96.1 & 18.6 \\
\hline Aovmartes por concepto de consulariat de $1+0$ & 225 & 5.1 & 224 & 43 \\
\hline Total Fuentes Internacionales & 100.2 & 24.9 & 118.5 & 22.0 \\
\hline Total & 438.1 & 100.0 & 517.5 & 100.0 \\
\hline
\end{tabular}

Fuente: I Censo Nacional de imestigación y Desamalis.
Elaboración: CONCYTEC - Oinaccion de irvestipacion y Estudios.

Al 2015, el 58.3\% del gasto en I+D se financió con fondos propios, mientras que el $18.8 \%$ fue financiado por fondos provenientes de terceros o concursables. Por último, el $22.9 \%$ se financió por fondos internacionales, a nivel agregado. 
Las investigaciones científicas que las universidades tanto públicas como privadas realizan, deberían estar enfocadas en el desarrollo social, es decir cubrir las necesidades primordiales de la sociedad en su conjunto, antes de crear tecnologías avanzadas que sólo se pueden aplicar en la capital y en las zonas urbanas del país.

Los presupuestos Públicos destinados para las investigaciones científicas, que están a cargo de la FONDECYT, deberían incentivar a que sólo las Universidades e Institutos públicos tengan la mayor preferencia ya que esos recursos volverán a la sociedad, mientras que las privadas, ya cuentan con la financiación de sus propias investigaciones y tienen las facilidades de poder desenvolverse en el sector empresarial porque las leyes las amparan.

\section{Universidades del Perú en el Ranking Scimago}

A nivel internacional el Perú se ubica en el puesto 72 en calidad de las instituciones dedicadas a la investigación, según el Informe Global de Competitividad 2017-2018 que evalúa los factores que impulsan la productividad y crecimiento en 137 países, lo cual representa un retroceso de 5 posiciones respecto al Informe del año pasado y 11 posiciones respecto al puesto 61 que ocupó el año 2013.

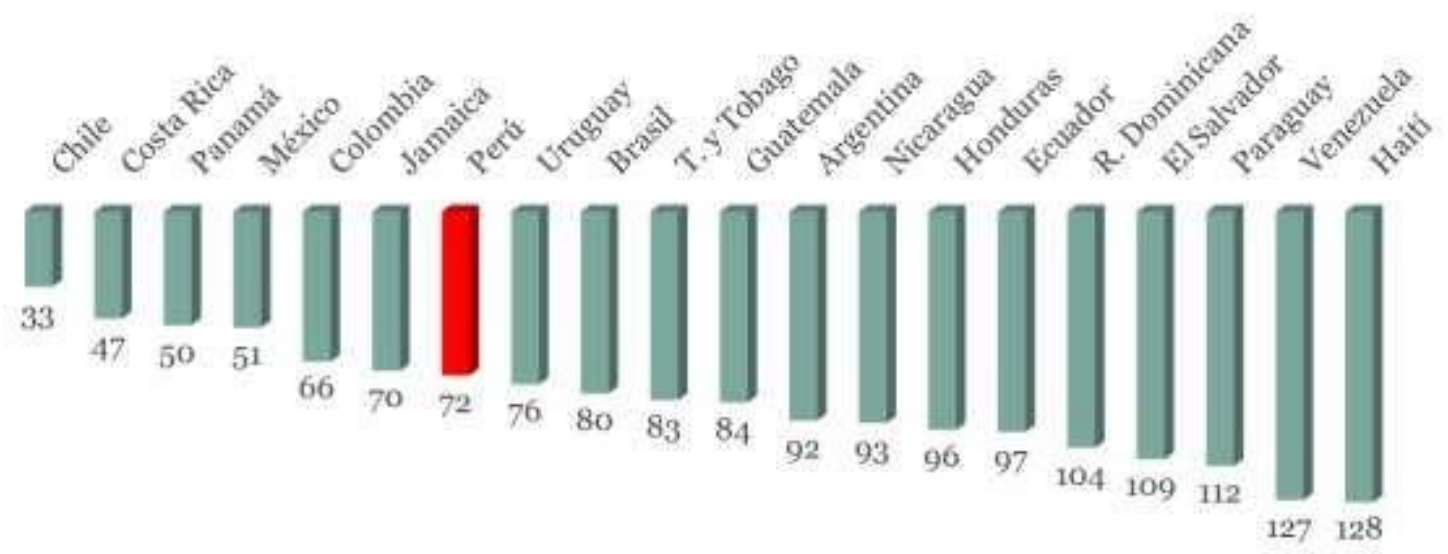

Fuente: http://www.cdi.org.pe/InformeGlobaldeCompetitividad/index.html

Un importante indicador que nos puede mostrar una idea del estado actual de investigación científica en las universidades del Perú tiene que ver con el número de producciones científicas desarrolladas. Según el grupo SIR- SCIMAGO en el 2014 el Perú se encontraba en el penúltimo lugar a nivel de lberoamérica.

El Ranking Scimago es el que mide los niveles de investigación científica, innovación y presencia en la web de las universidades de todo el mundo. Según este estudio el Perú se encuentra penúltimo a nivel de Iberoamérica, con solo dos universidades (contra 75 de Brasil por ejemplo, o 14 de Chile) que se encuentran en estas condiciones: la Universidad Peruana Cayetano Heredia y la Universidad Nacional Mayor de San Marcos, según el mismo informe de Contraloría.

En junio del 2017, el ranking Scimago Institutions Rankings (SIR) consideró a cuatro universidades peruanas con el mayor promedio ponderado de investigaciones científicas: la Pontificia Universidad Católica del Perú (PUCP), la Universidad Peruana Cayetano Heredia (UPCH), la Universidad Peruana de Ciencias Aplicadas (UPC) y la Universidad Nacional Mayor de San Marcos (UNMSM).

La PUCP se ubica en el primer lugar, pues tiene más de cien proyectos en marcha, a pesar que en el rubro social, no se toma en cuenta los alcances en responsabilidad social, que es un punto fuerte de la PUCP. Los resultados de los últimos cinco años arrojan un crecimiento significativo en materia de investigación para la PUCP. Esto coincide con la política institucional de apoyo a la investigación que trajo consigo la creación del Vicerrectorado de Investigación (VRI) y la Dirección de Gestión de la Investigación (DGI) en el 2009.

En segundo lugar, se encuentra la UPCH que, de acuerdo con el Ranking Iberoamericano SIR 2012, ocupaba primer lugar entre las instituciones peruanas en producción científica, en vista que la política 
institucional de la UPCH promueve la investigación y la formación de recursos humanos para la investigación. Cabe mencionar que la UPCH presentó, el pasado 10 de junio, su Catálogo de Laboratorios, Unidades, Centros e Institutos de Investigación 2016-2017 en la Casa Honorio Delgado de Miraflores, con la presencia de importantes personalidades del rubro empresarial y científico del país, además Cayetano es la primera universidad acreditada que desarrolla investigación en las áreas de ciencias de la vida. Produce el 33\% de las publicaciones científicas del país. La Universidad Peruana Cayetano Heredia (UPCH), se dedica a investigaciones sobre enfermedades de altura y enfermedades tropicales, y el Instituto Geofísico del Perú (IGP), dedicado a investigaciones sobre la ionósfera. Según sus directivos, este éxito se debe, en gran parte, al financiamiento que reciben de las instituciones internacionales que costean las investigaciones que se encuentran en sus rubros de interés.

Así también, en el reciente ránking $Q S$, se le ha reconocido entre las 4 universidades que más investigación hacen en Sudamérica, Informe 2014 - las 10

universidades peruanas con mayor producción científica, según el último informe 2014 SCImago Research Group, encargado de evaluar y analizar la producción científica de las instituciones de educación superior, la Cayetano Heredia es la universidad peruana con mayor producción científica (1089 publicaciones) entre 2008 y 2012. Ninguna universidad peruana figura entre las 100 primeras de Iberoamérica.

En tercer lugar encontramos a la UPC que desde agosto del 2014 viene destacando en La Revista Digital de Investigación en Docencia Universitaria (RIDU), elaborada por el Área de Investigación de la Dirección de Calidad Educativa de la Universidad Peruana de Ciencias Aplicadas (UPC); se ha convertido en la primera revista de investigación y docencia universitaria del país que ha sido indexada en Latindex, tras dos años de arduo trabajo y exigencia. Este catálogo internacional es un sistema de información sobre las revistas de investigación científica, técnico-profesionales y de divulgación científica y cultural, que se editan en los países de América Latina, el Caribe, España y Portugal. Hasta el momento, RIDU tiene siete ediciones, las cuales son publicadas en el mes de diciembre.

Finalmente, en el cuarto lugar se encuentra la decana de América la UNMSM descollando también en el último ránking de universidades de América Latina, creado por la consultora británica QS. Tiene tan solo una sola universidad pública entre las 100 mejores, la Universidad Nacional Mayor de San Marcos (UNMSM) situada en el puesto 60. La UNMSM en el año 2013, se manifestó que las áreas con mayor número de proyectos corresponden a las Ciencias Básicas, Ciencias de la Salud e Ingenierías. Según el Consejo Superior de Investigación de la UNMSM, San Marcos lideró la lista de universidades con más revistas indizadas en el 2014. Según Latindex, Sistema Regional de Información en Línea para Revistas Científicas, con presencia en 32 países de Hispanoamérica, San Marcos es la universidad que tiene más revistas científicas indizadas. El procedimiento comienza con las universidades peruanas enviando sus revistas al Consejo Nacional de Ciencia, Tecnología e Innovación Tecnológica (CONCYTEC) para que sean evaluadas. Es CONCYTEC quien las registra en Latindex.

En el Perú, hay a la fecha 99 revistas indizadas editadas por universidades. San Marcos lidera la lista con 15, sigue la PUCP con 13 según Ranking Scimago Iberoamérica 2015: En Perú no hay novedades entre las primeras pero UPC mejora. España sigue liderando pero Latinoamérica se acerca.

\section{Planes de estudio}

Otra problemática que detectamos se encuentra a nivel de los planes de estudio de una carrera universitaria, porque nos muestra la tendencia, no solo en cuanto a la materia que se estudia, sino al tipo de profesional que se pretende formar. Este tipo de profesional, generalmente, está definido en el Perfil del Profesional de cada carrera. Al leer estos perfiles notamos que pocos de ellos ponen énfasis en la investigación, así como la gran variedad de cursos que presentan la misma carrera en dos universidades. Analizamos 12 planes de estudios y notamos que para graduarse se llevan en promedio 55 cursos durante los 5 años de estudios. Constatamos que no más del 10\% de los cursos que se imparten durante una carrera de 5 años, hacen alguna referencia expresa a la investigación. Esto equivale a un máximo de 6 cursos, es el caso de Estomatología de la UPCH; y un mínimo de 2 cursos, en el caso de Química de la UNMSM. 
Entonces nos preguntamos, ¿'son suficientes esos cursos para formar investigadores? Para responder esta pregunta quizás valga esta comparación: si se considera que el egresado, a pesar de haber aprobado los cerca de 55 cursos de su carrera, egresa de la universidad sabiendo que necesita conocer más para desempeñarse con eficiencia en su profesión, entonces, ¿con 5 o 6 cursos, se puede formar a un investigador? La respuesta es, claramente, no.

Por otro lado, si analizamos el contenido de los cursos, encontramos que, la mayoría de ellos son similares, más que en contenido, en su carácter meramente informativo de un tópico. Exponen de manera muy romántica, y muchas veces rimbombante, lo que es ciencia, investigación, y/o presentan la estructura de una investigación, pero, no dicen cómo hacerlo. Es más, algunos comienzan con el proyecto de tesis y esperan que el estudiante lo desarrolle presuponiendo que ya conoce qué es y cómo se desarrolla cada capítulo.

Así, muchos de los cursos que observamos proveen de información al estudiante, de información que puede encontrarse con mucha facilidad hoy en día por toda la red. Pero, ¿es inteligente centrarse en dar información en este mundo cada vez más "infoxicado"? es decir, saturado de información. Creemos que no. Consideramos que los profesores deben enfocarse en adiestrar o entrenar en el análisis crítico, la comparación, en la selección de datos, la organización de los mismos, la elaboración conceptual, la argumentación, la redacción científica, las referencias bibliográficas, etc.

El problema descrito aquí es, sin duda, consecuencia de la formación con mallas curriculares que contienen cursos que ponen énfasis en la presentación de datos y más datos. Al parecer, la concepción de la universidad ha decaído a fuente de datos y el profesor es ahora un datero. Con innegable contradicción, el estudiante egresa sin capacitación ni destrezas suficientes para la investigación. Solo para la recopilación de información. Esto lo notamos cuando el graduando intenta desarrollar su tesis de licenciatura o de maestría.

A pesar que la nueva Ley Universitaria exige el desarrollo de dos tesis: una para bachiller otra para licenciatura, medida adecuada para incrementar el número de investigaciones, sin embargo creemos que si no se cambian los planes de estudio o no se trabaja en un diseño curricular enfocado en competencias, formación de capacidades y destrezas para la investigación, los graduandos seguirán recurriendo a los quioscos para comprar trabajos sin ningún valor académico nada claro, ni ético.

\section{Conclusiones}

Creemos que el pensamiento de José Carlos Mariátegui, se hace vigente en la problemática estudiada: "No se puede democratizar la educación, sino se democratiza la economía", porque todos los críticos, estudiosos de la realidad nacional, incluidos nosotros, somos conscientes que no se puede hacer investigación sino contamos con los presupuestos reales y dignos que requiere la investigación universitaria. En la política de incentivos económicos debemos imitar las acciones que realizan los países desarrollados.

Dentro de los desafíos que debe enfrentar el sistema universitario se encuentra los procesos de licenciamiento y acreditación en donde se exige cumplir estándares mínimos para ofertar una verdadera calidad educativa, no obstante, se exige, pero no se asignan los presupuestos para optimizar la infraestructura, los laboratorios y talleres y contratar y nombrar docentes idóneos de perfil investigativo. A raíz del primer problema de índole económico, las universidades no cumplen su rol de investigación, y si éstas se realizan, se brinda más apoyo a las llamadas ciencias naturales o duras como la física, la química, la biología, la matemática y se subestiman a las llamadas ciencias blandas, en especial las ciencias sociales y las humanidades. La situación real de nuestro país nos debe inclinar hacia el desarrollo de la gestión humana que contribuya realmente a la solución de los problemas sociales.

En la falta de una cultura investigativa están involucrados los docentes por la escasa o limitada capacitación que se les brinda o que ellos mismos no lo asumen. Se les asigna cursos de investigación pero su desarrollo adolece de la falta de estrategias, metodologías y recursos para la elaboración de proyectos. Asimismo, los planes de estudio de las universidades peruanas no están diseñados para formar 
investigadores, contienen cursos en la que solo se presenta información relacionada con la disciplina pero desconectada de un propósito de investigación.

Las políticas que se están aplicando y que generan un escenario de preocupación, por así decirlo, para poder ser parte como muchos países a la OCDE, trae como consecuencia que se estén priorizando proyectos de investigación de corte tecnológica, asimismo, los programas de maestrías y doctorados van también por esa vía; y los recursos destinados para el financiamiento de esos proyectos de investigación, se destinen en un gran porcentaje a las universidades o institutos que puedan desarrollar esos proyectos. Por los tanto, las universidades públicas quedan relegadas de participar, o si lo hacen son pocas, ya que sus laboratorios no cuentan con las herramientas necesarias para desarrollar esos proyectos.

\section{Bibliografia}

Caballero, C. (2017), Cinco ideas falsas de las humanidades. El Búho Diario digital. Recuperado de http://elbuho.pe/2017/09/19/cinco-ideas-falsaslas-humanidades/

CONCYTEC (2016), Política nacional para el desarrollo de la ciencia, tecnología e innovación tecnológica -CTI. Recuperado de https://portal.concytec.gob.pe/images/documentos/Politica_Nacional_CTI2016.pdf

Dávila, D. (2011), Los que nos dicen los planes de estudio. Ciencia.pe. Recuperado de http://ciencias.pe/opinión/ ¿por-qué-se-investiga-tanpoco-en-las-universidades-peruanas

Garfias M. (2011), La investigación en la universidad pública regional y los fondos del canon, 2004-2008. Lima: IEP.

Purizaca-Rosillo, Nelson, Cardoza-Jiménez, Kennlly, \& Herrera-Añazco, Percy (2016), Scientific production in a Peruvian public university beneficiary of canon. Anales de la Facultad de Medicina, 77(1), pp. 73-74. https://dx.doi.org/10.15381/anales.v77i1.11561

\section{Otras fuentes online:}

http://www.cdi.org.pe/InformeGlobaldeCompetitividad/index.html

http://investigacion.pucp.edu.pe/investigacion/la-pucp-ocupa-primer-lugar-nacionalscimagoinstitutions-rankings-sir-2017/

http://www.upch.edu.pe/portal/ver-mas/item/2502-cayetano-lanzo-su-catalogo-deinvestigacion-20162017.html

http://revistas.upc.edu.pe/index.php/docencia/index 